\title{
Making Concept Maps Available on the Web to the Students
}

Sandra Lopes, Pedro Henriques, Paulo Dias

Universidade do Minho

4700 Braga, Portugal

sandralopes@di.uminho.pt

\section{Introduction}

Nowadays, the web sites that most of us make available to the students, to support our courses, are mere transposition of the old article material to the computer. This is, our course web sites are just repositories of links and disconnected chunks of descriptions-objectives and course structure, learning outcomes, program (table of contents, or topic index), bibliography and other supporting materials, assessment and marking rules, teaching team, classes and timetables, etc. The interaction of the students with the site and its relevance for the knowledge acquisition process are minimal. This is particularly upsetting when we teach computer science students or we teach computers to other students (engineering, social sciences, etc.)-the best way to convince them of the computer capabilities is to apply it in our own profit. Indeed, teaching how to solve our daily problems with computers, and being unable to take the best of them in our activities, is contradictory.

So, we argue that it is mandatory to transform the web site into a learning object, being an effective aid for students to understand the course subject and acquire new knowledge on that area. Different constituents of the site-like the programme (the index of course units), lesson synopsis and summaries, bibliography, objectives, etc.-should be strongly related in such a manner that students can go through them in a kind of a semantic navigation; In this way, we believe that it can contribute to their learning 
process. In the article, we suggest the use of Concept Maps to support that transformation.

We advocate that the course planning should be done based on a Concept Map representing the knowledge domain involved. After the planning, this Concept Map should be kept and made available to the students. To be useable, a computer-based tool should support the Concept Maps development and navigation.

In this context and with that motivation in mind, this article aims at introducing a system, NAVMAP, to help teachers create a navigator for Decorated Concept Maps as the result of their course planning activity.

The article is structured as follows. We remember the definition of Concept Map and its role in the course planning process, in Section 2. Then, Section 3 is devoted to NAVMAP; after an overview of the system functionality, we present NAVMAP architecture and detail its two main components: the Decorated Concept Maps Navigator; and the information management sub-system that assists the course planning activity. Section 4 is a short discussion on the benefits of using NAVMAP to build a learning object linkable to a course web site, and Section 5 is the conclusion.

\section{Concept Maps and Planning}

This section is to introduce the role of Concept Maps in the teaching planning activity (PA).

Concept mapping is a technique for representing knowledge in graphs. Knowledge graphs are networks of concepts. A graph consists of nodes and arcs. Nodes represent concepts and links represent the relations between concepts (see Figure 1 that shows a partial Concept Map taken from our course on Introduction to Computers). The Concept Maps may be translated in propositions. Propositions are obtained by going through the graph, reading the substantives (concepts) inscribed in the nodes concatenated to the transitive verbs (relationships) labelling the arcs.

The use of Concept Maps as a teaching strategy was first developed by Novak in the early 1980's [1]. It was derived from Ausubel's learning theory [4] that places central emphasis on the influence of students' prior knowledge on subsequent meaningful learning. According to Ausubel, "the most important single factor influencing learning is what the learner already knows. Thus meaningful learning results when a person consciously and explicitly ties new knowledge to relevant concepts they already possess". Ausubel suggests that when meaningful learning occurs, it produces a series of changes within our entire cognitive structure, modifying existing concepts and forming new linkages between concepts. 


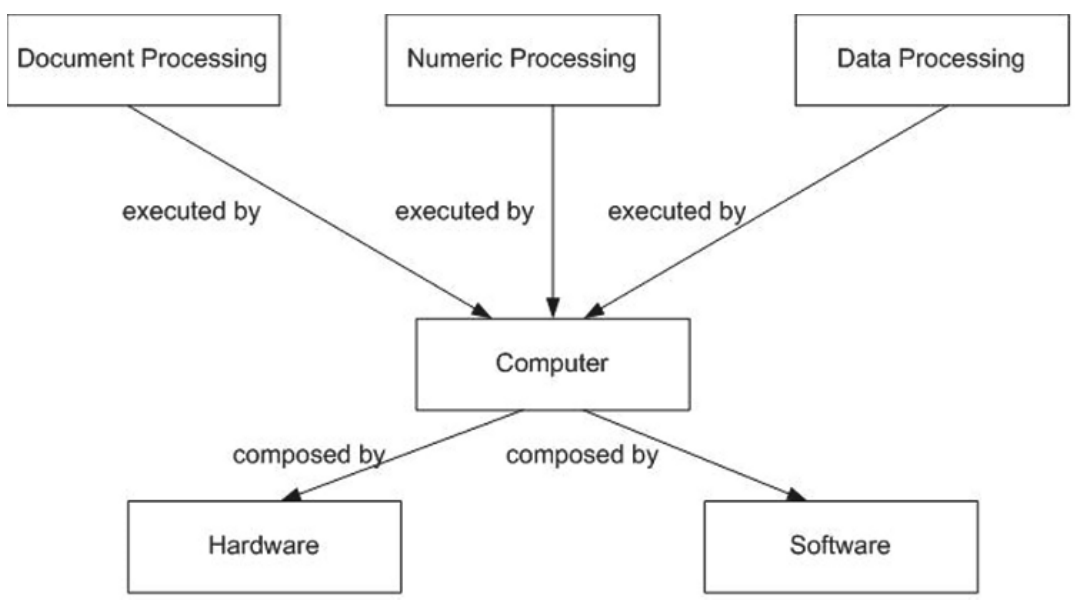

Fig. 1. Concept Map of a course planning

Concept Maps is, also, considered a pedagogical strategy for planning; because it allows organizing the course contents by concepts to be taught. The wide and integrating concepts may constitute the basis of planning while the less embracing concepts are used to select the most specific teaching activities and information resources.

The planning process can be split into four steps: definition of the concepts to be taught; organization the concept network; association of concepts to teaching units and scheduling (distribution of units amongst the course's available time); and choice of resources to use associating them to concepts [8].

However, building and handling Concept Maps is a complex and time consuming task either for the teachers, or for the students to use them as learning object. Therefore, there is a strong need for a computer based system able to process Concept Maps (edit, store, and navigate); for that a rigorous representation system is necessary.

\section{NAVMAP}

NAVMAP is a web application supported by a database to:

- Define Concepts and Relations;

- Associate Concepts and Relations, creating a true and traditional Concept Map (CM);

- Define course Units, associating them to Lessons;

- Associate Concepts to course Units, producing a Coloured Concept Map (CCM); 
- Define Information Resources;

- Associate Concepts to Resources, creating a Decorated Concept Map (DCM);

- Navigate over the Decorated Concept Map.

Figure 2 shows the complete workflow to produce a Decorated Concept Map Navigator. NAVMAP also allows the user to export the Decorated Concept Map to a standard format, XTM (XML Topic Map), to interchange the map data with external applications. The purpose of a Topic Map (TM) is to convey knowledge about resources through a superimposed layer, or map, of the resources. A topic map captures the subjects of which resources speak, and the relationships between subjects, in a way that is implementation-independent [7]. The key concepts in topic maps

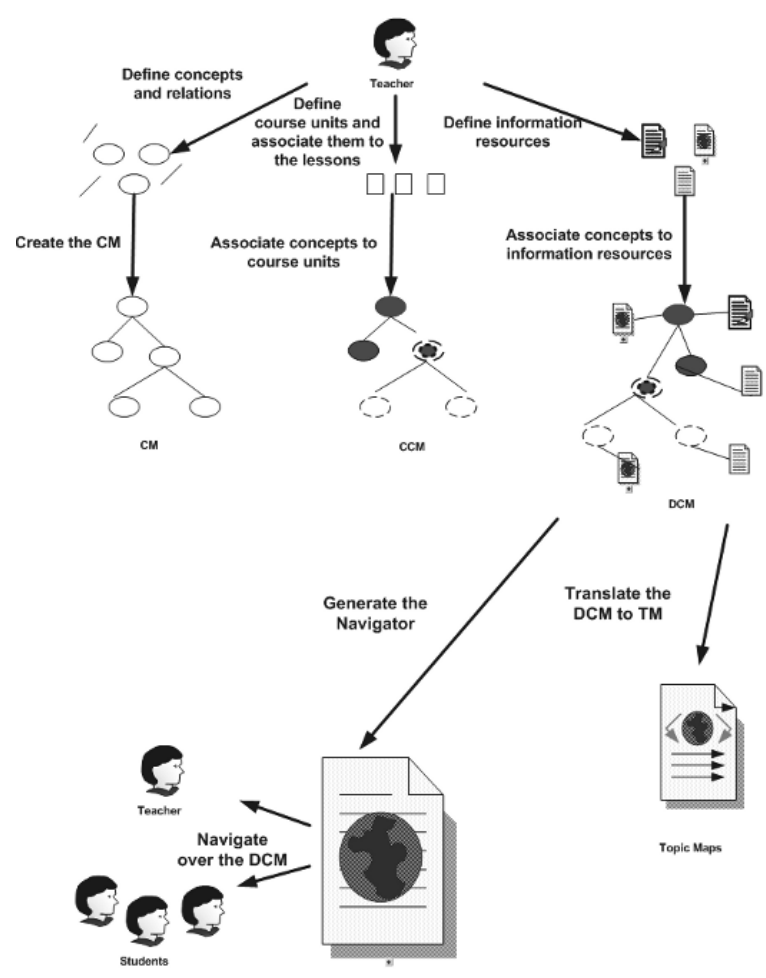

Fig. 2. Workflow supported by NAVMAP

are topics, associations, and occurrences; so Concept Maps can be directly translated to Topic Maps as discussed in [6]. This feature enables the use 
of other tools that handle that representation (TM) for semantic networks. For instance, we can use the navigator generators Ulisses [3] or Omnigator [5]; or the Concept Maps processor CmapTools [2].

NAVMAP allows exploring course-planning material in three different ways:

- Navigating through the concepts;

- Navigating through the course units;

- Navigating through the information resources.

To accomplish its task, two parts compose NAVMAP: the Back-Office (BO) that supports the teachers' planning, assisting him in the development of the Decorated Concept Map; the Front-Office (FO), available to everyone (teachers and students), to navigate over the Decorated Concept Map.

\subsection{NAVMAP Architecture}

The two main components of NAVMAP architecture (FO and BO) are supported by a central database, as depicted in Figure 3. The BO is

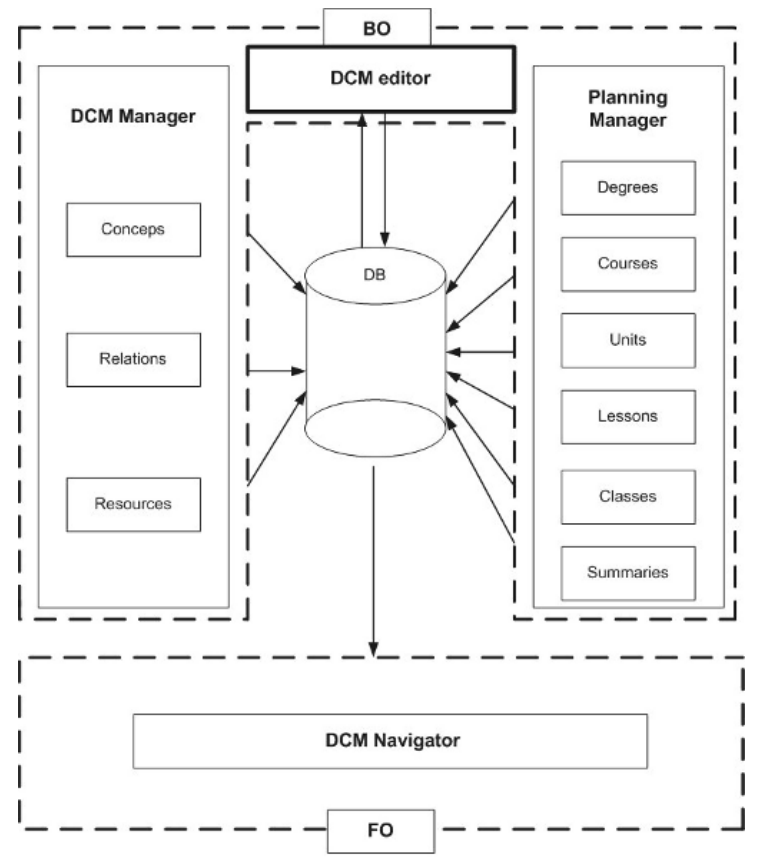

Fig. 3. Architecture of NAVMAP system 
responsible for storing and keeping the information in the central database, and can be splitted into three parts:

1. A DCM Manager that handles concepts, relations and resources;

2. A Concept Maps Editor and Integrator with Course Units and Information Resources (DCM Editor in figure 3.);

3. A Planning Manager that handles degrees, courses, units, lessons, classes and summaries;

The Front-Office just queries the central database and dynamically generates the HTML pages to visualize and traverse the conceptual network.

\subsection{NAVMAP: The Navigator}

Figure 4 is a screen of the navigator produced by NAVMAP Front-Office for the Concept Map shown in Figure 1. The page shown is a specific view corresponding to the selection of one particular concept ("Computer"). The selected concept is displayed at the centre of the page; the related concepts are shown around it (in this case there are five concepts). The arrows connecting concepts, three in-coming and two out-coming, are labelled by the name of the relations-observing that fragment of the Concept Map, we can say that "Document Processing" is "executed by" "Computer", and

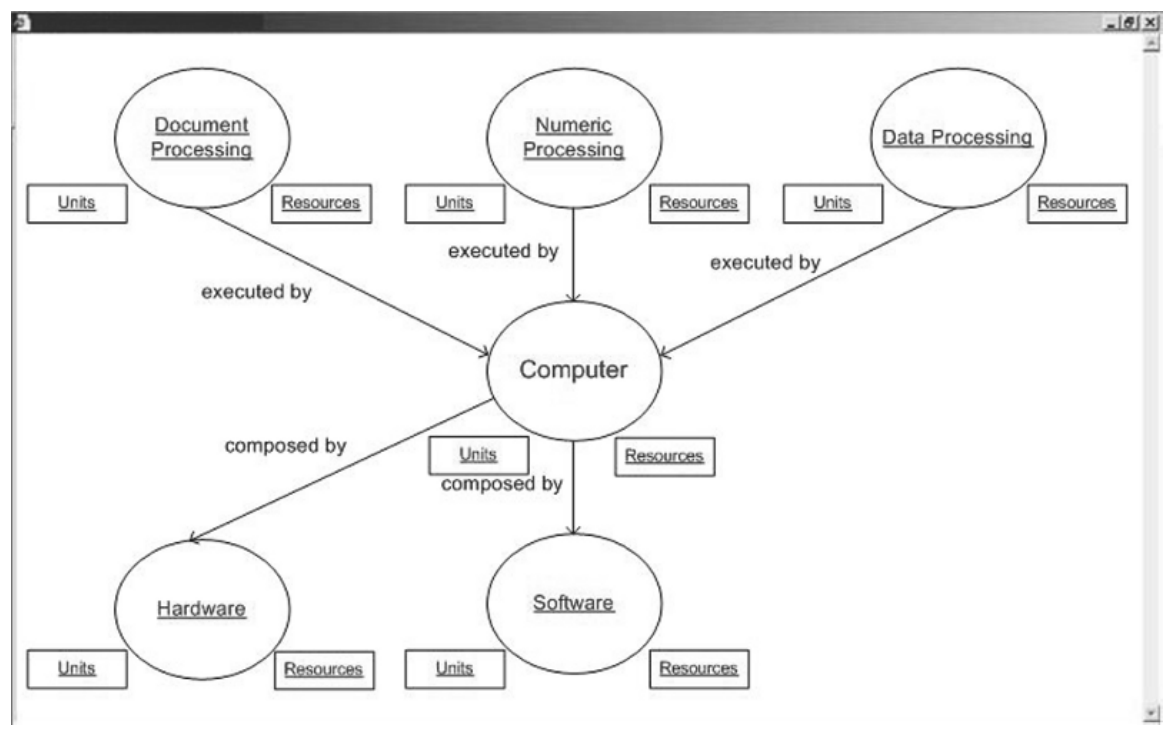

Fig. 4. Screen of DCM Navigator 
that "Computer" is "composed by" "Hardware". Units and Resources associated with each Concept are represented, displaying their names inside rectangles locate below the respective node; units on the left and resources on the right.

When we invoke the Navigator, the "Concept Map root" is selected and displayed at the centre of the first page; by default, we assume the root as the concept whose name is the course name.

The student can interact with the page in order to navigate over the Decorated Concept Map; all that he has to do is to follow the link associated with the name of one of the concepts, units or resources, depending on the kind of exploration he intends to do (as previously told, the user can go through concepts, course units where concepts are taught, or information resources proposed to support each concept).

\subsection{NAVMAP: The Planning support}

The most important characteristic of the NAVMAP Back-Office (BO) is that all the insertion and editing operations are done in textual forms, instead of adopting a visual (or graphical) approach.

To illustrate the BO that the teacher uses to build and decorated the Concept Map (creating and updating the database that is searched by the Front-Office to generate the desired navigator), we show some screenshots of the web application.

Figure 5 is the form for adding course units. The degree and course names, to which the unit belongs, are initially defined and appear at the top of the page. As can be seen, the units already defined are listed inside a

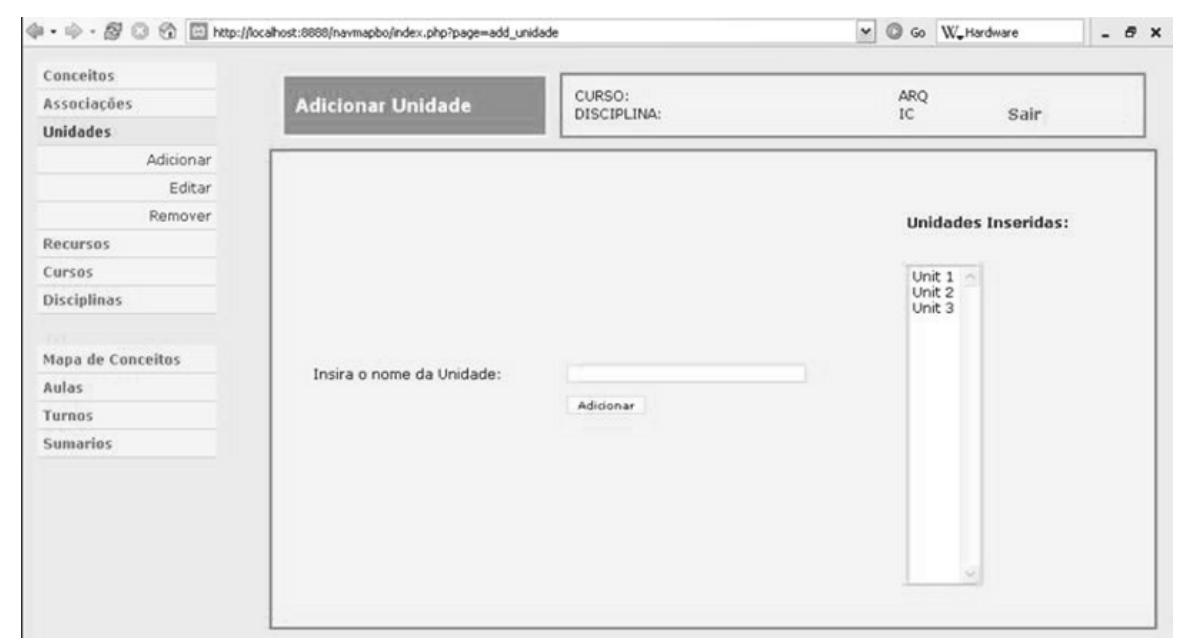

Fig. 5. Interface of the Planning Manager (course Units) 
box on the right of the screen; just the unit name must the provided. Another different option should be selected to associate units to lessons, and lessons synopsis and summaries.

Figure 6 is the form to edit concepts. The concepts previously inserted are displayed in the list box on the left; the user can select the one he intends to edit, and then update the information shown inside the boxes on the right.

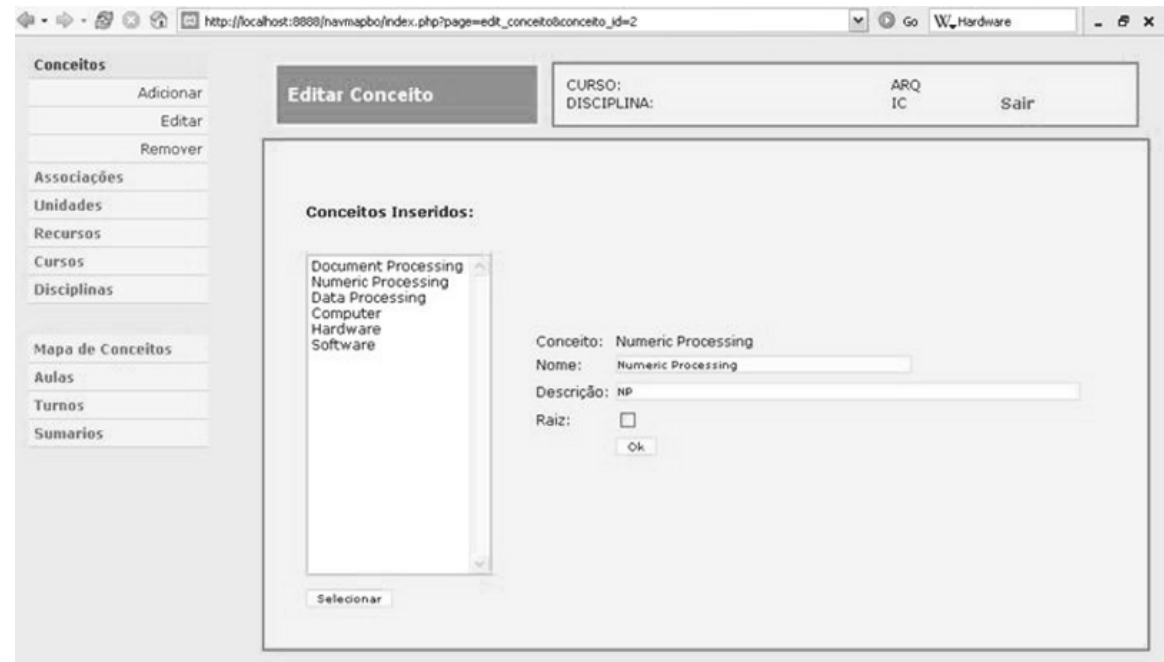

Fig. 6. Interface of DCM Manager (Concepts)

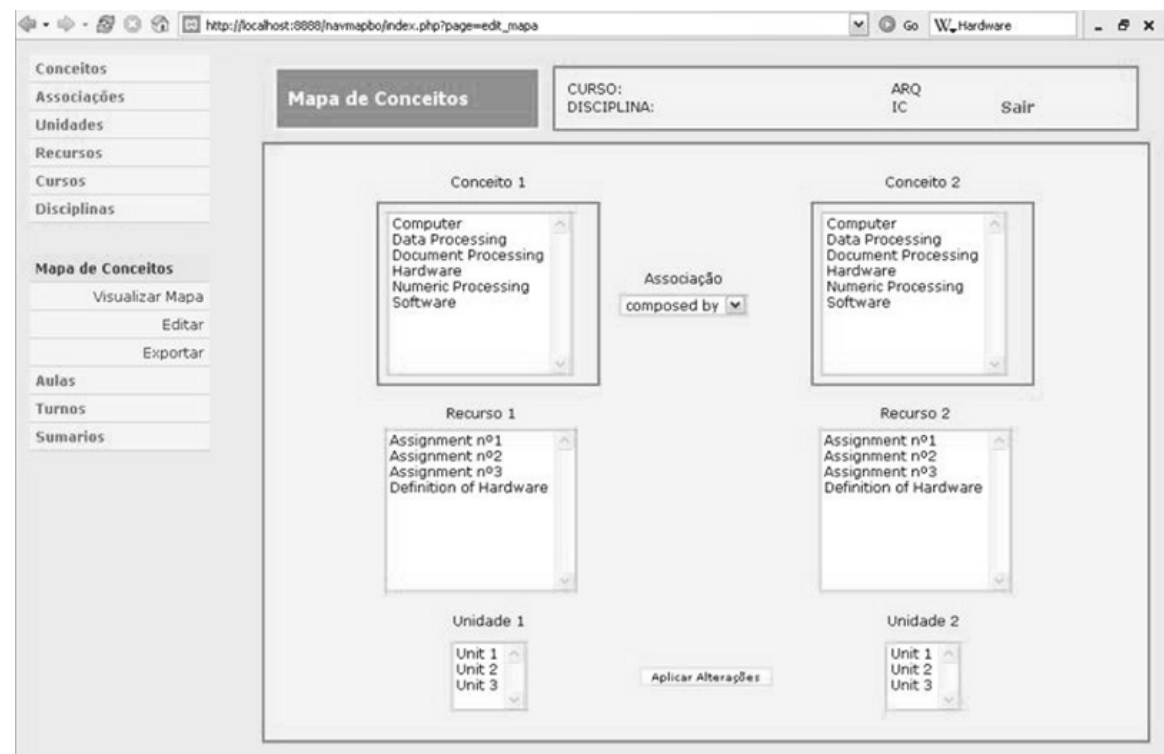

Fig. 7. Interface of Decorated Concept Maps Editor 
The form to add/edit resources is quite similar to the previous ones; however, a search facility is included.

After adding the basic components, what should be done is the association of concepts by means of relations, and their connection to units and resources; this task is executed fulfilling the form shown in figure 7.

\section{The Outcomes of NAVMAP}

In this section we will discuss the pedagogical outcomes we hope to attain with the use of our system in the classroom.

From the teacher's point of view, NAVMAP makes the Planning Activity a simpler and a more systematic process. The edition of the Concept Map and its maintenance becomes much easier, as well as its association with the course units, lessons, and resources.

From the students' point of view, a great advantage of using NAVMAP is that it provides a visual representation of the Concept Map under study in a tangible form that can be remembered very easily. The Concept Map can be readily revisited as many times as necessary. During the navigation the students understands perfectly the concepts' meaning and the relationships established among them.

One general benefit is that course planning becomes useful for both, teachers and students. It is no more just an administrative instrument to exhibit in the first lesson and archive thereafter.

Other general benefit is that the course site is transformed into a learning object and not only a repository of information.

\section{Conclusion}

To plan a course, the teacher should start by defining the abilities and skills that the students will acquire after following the lessons; in other words, he should specify the learning outcomes. An effective way to proceed, in order to define the course contents and to draw up the course programme is to develop a Concepts Map, in the sense of Ausubel and Novak. However, that task is hard to accomplish and, most of the times, it has not any impact in the teaching/learning process.

We advocate that the Concept Map should be made available to the students, on one hand to improve their understanding about the topics under study, and on the other hand to aid the assessment process (by comparing the student Concept Map against the teacher Concept Map). 
In that context, this article discussed the approach and introduced NAVMAP, a web tool to help teachers' planning and to produce a navigator that can be added to the course web site for students' usage. In this way, we believe that a computer science teacher is releasing a much more interactive and up-to-date tool; his course web site is no more just a collection of links, but offers a semantic network of concepts.

The next step is to merge different Concept Maps, allowing an academic manager to get a general overview of a set of courses (belonging to some degree). Such a feature enables the manager to understand where and how some subjects are introduced and discussed, allowing him to balance the institutional teaching offer. This new functionality can be achieved either developing a new module that queries the central database, or using a special tool to merge Concept Maps.

\section{References}

1. Ausubel D (2000) The Acquisition and Retention of Knowledge: A Cognitive View. Kluwer Academic Publishers, Boston

2. CmapTools, http://cmap.ihmc.us/

3. Librelotto GR, Ramalho JC, Henriques PR (2003) Ontology driven Websites with Topic Maps. In The International Conference on Web Engineering, Oviedo, Spain.

4. Novak J, Gowin D (1984) Learning How to Learn. Cambridge University Press, New York

5. Ontopia. The Ontopia Knowledge Suite. http://www.ontopia.net/solutions/ products.html.-

6. Paz F, Lopes SC, Librelotto GR, Henriques PR (2005) XML Topic Maps e Mapa de Conceitos. XATA' $05-3^{\mathrm{a} C}$ Conferência Nacional sobre "XML Aplicações e Tecnologias Associadas", Braga

7. Pepper S (2000) The TAO of Topic Maps. In proceedings of the XML Europe 2000.

8. Ribeiro AC, Ribeiro LC (2003) Planificação e Avaliação do EnsinoAprendizagem. Universidade Aberta, Lisboa 